
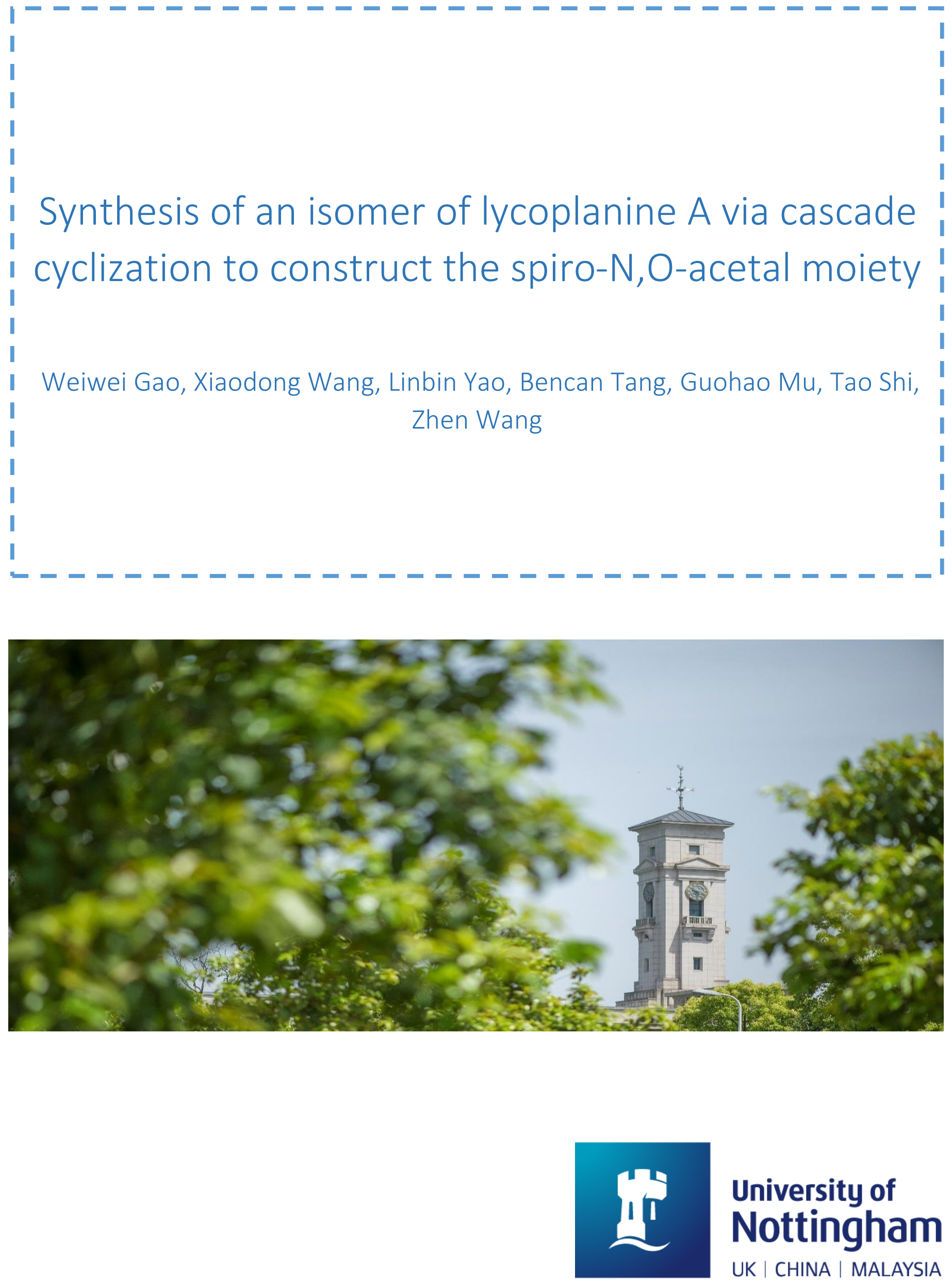
University of Nottingham Ningbo China, 199 Taikang East Road, Ningbo, 315100, Zhejiang, China.

First published 2021

This work is made available under the terms of the Creative Commons Attribution 4.0 International License:

http://creativecommons.org/licenses/by/4.0

The work is licenced to the University of Nottingham Ningbo China under the Global University Publication Licence:

https://www.nottingham.edu.cn/en/library/documents/researchsupport/global-university-publications-licence-2.0.pdf 


\section{Synthesis of an isomer of lycoplanine A via cascade cyclization to construct the spiro-N,O-acetal moiety}

Received 00th January 20xx, Accepted 00th January 20xx

\author{
Weiwei Gao, ${ }^{+\mathrm{a}}$ Xiaodong Wang, ${ }^{\mathrm{b}}$ Linbin Yao, ${ }^{+\mathrm{c}}$ Bencan Tang, ${ }^{\mathrm{c}}$ Guohao Mu, ${ }^{\mathrm{c}}$ Tao Shi*b and Zhen \\ Wang*a,b
}

DOI: $10.1039 / \times 0 \times x 00000 x$

An isomer of lycoplanine A with a 6/10/5/5 tetracyclic skeleton was synthesized using D-A reaction and cascasde reaction to respectively construct the [9.2.2] pentadecane skeleton and the challenging 1-oxa-6-azaspiro[4.4]nonane spirocenter. Morever, detailed DFT calculations were conducted to explain the selectivity in the D-A reaction. This study may provide sufficient experience for the total synthesis of lycoplanine $A$ and other alkaloids with similar spiro-N,O-acetal cores.

Lycopodium alkaloids, especially Huperzine A have shown great potential on the clinical treatment of Alzheimer's disease. ${ }^{1-2}$ Up to now, over 300 Lycopodium alkaloids have been isolated, ${ }^{3}$ and many beautiful total syntheses towards these alkaloids have been reported. ${ }^{4}$ In 2017 , lycoplanine A, a new type of Lycopodium alkaloid with a 6/9/5 tricyclic skeleton that is fused with the $\gamma$-lactone ring was firstly isolated and characterized by Zhao and co-workers. ${ }^{5}$ Notably, in lycoplanine A, an unusual 1-oxa-6-azaspiro[4.4]nonane moiety is embedded, which is usually a core of bioactive natural products ${ }^{6}$ and is generally obtained from oxidative spirocyclization of furan derivatives. ${ }^{7}$ Biological studies show that lycoplanine $A$ is a potent $\mathrm{Ca}_{\mathrm{v}} 3.1 \mathrm{~T}$-type calcium channel (TTCC) inhibitor with the $\mathrm{IC}_{50}$ value of $6.06 \mu \mathrm{M} .{ }^{5}$ In view of its unique structure and important biological activity, we herein explored the synthetic route to lycoplanine $A$, studied the rapid construction of the spiro- $\mathrm{N}, \mathrm{O}-$ acetal moiety in lycoplanine $\mathrm{A}$ and accomplished the synthesis of an isomer of lycoplanine A with a 6/10/5/5 tetracyclic skeleton.

From the perspective of structure, the $C$ and $D$ rings in lycoplanine A (1) could be disassembled via an efficient

a. State Key Laboratory of Applied Organic Chemistry, College of Chemistry and Chemical Engineering, Lanzhou University, Lanzhou 730000, China.

E-mail: zhenw@lzu.edu.cn

${ }^{b}$ School of Pharmacy, Lanzhou University, No. 199 West Donggang Road, Lanzhou 730000, China.E-mail: zhenw@lzu.edu.cn; shit18@Izu.edu.cn

c Department of Chemical and Environmental Engineering, Faculty of Science and Engineering, The University of Nottingham Ningbo China, 199 Taikang East Road, Ningbo, 315100, China.

+ These authors contributed equally to this work.

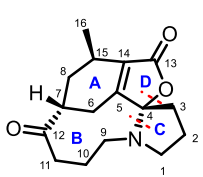

1: Lycoplanine A

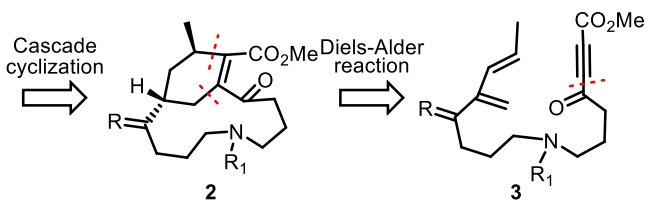

Scheme 1 Retrosynthetic analysis of lycoplanine A.

tandem cyclization initiated from N-protected compound 2, referring to the proposed biogenetic pathway between lycopladine $\mathrm{H}$ and lycoplanine A reported by Zhao et al. (Scheme 1). ${ }^{5}$ The six-membered ring in $\mathbf{2}$ is proposed to be dissociated via intramolecular Diels-Alder reaction of $3 .^{8}$ The key to synthesize D-A precursor $\mathbf{3}$ is the assembly of diene and dienophiles moieties.

With the above retrosynthetic analysis in mind, we started our synthesis via sequential installation of the diene and dienophile sections in D-A precursor 3 (Scheme 2). Starting from 1,3-dithiane 4, the first $\mathrm{C}=\mathrm{C}$ bond was effectively introduced employing Corey-Seebach reaction with the commercially available crotonaldehyde ( $E / Z>98 \%)$, giving alcohol 5 in $88 \%$ yield. ${ }^{9}$ Sequentially, alcohol $\mathbf{5}$ was oxidized with Dess-Martin periodinane to deliver ketone 6 in $80 \%$ yield. To introduce the second $\mathrm{C}=\mathrm{C}$ bond of $\mathrm{D}-\mathrm{A}$ precursor $\mathbf{3}$, numerous efforts were devoted, including Wittig reaction, Tebbe's reagent, and Nysted reagent. Only Nysted reagent gave methylenated product $\mathbf{7}$ in $63 \%$ yield. ${ }^{10}$ At this stage, the diene moiety of D-A precursor 3 has been assembled, then attention was turned to the introduction of the dienophile moiety. Deprotection of the silane protecting group by TBAF and a typical Mitsunobu reaction with fragment $A$ were conducted to lengthen the carbon chain as well as introduce the nitrogen atom, affording o-nitrobenzene sulfonyl protected amine $8 .^{11}$ Before the latestage introduction of the dienophile moiety, protecting group transformation of nitrogen atom from $\mathrm{o}-\mathrm{Ns}$ to $\mathrm{Boc}$ and the ensuing deprotection of hydroxy group were performed to give alcohol 9. After oxidation of the primary alcohol 9, the in-situ generated anion of methyl propiolate was added onto the aldehyde moiety to deliver alcohol 10, achieving the introduction of the dienophile section. Considering that alcohol 10 has possessed necessary structural features for D-A reaction, 

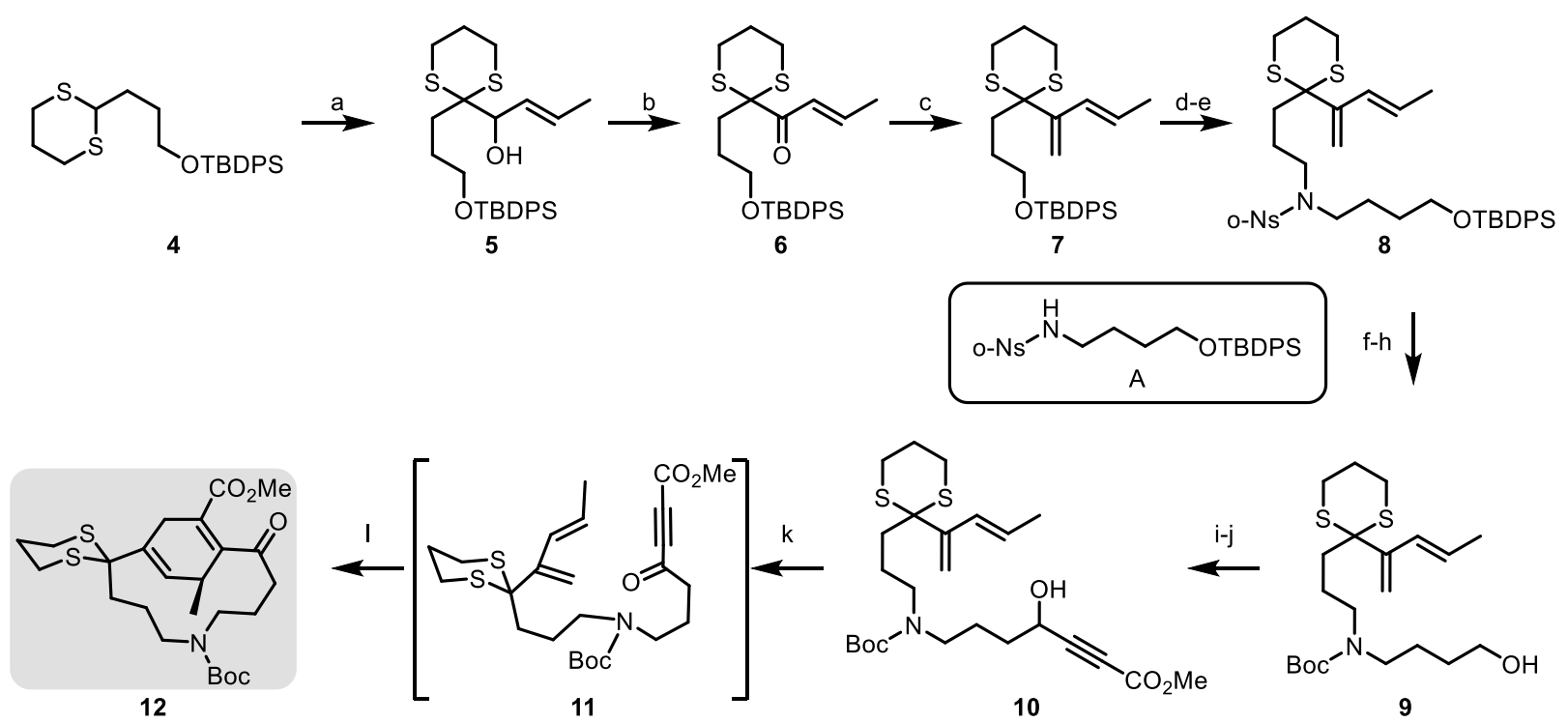

Scheme 2 Synthetic route to the Diels-Alder Product 16. Reagents and conditions: (a) $t$ - $\mathrm{BuLi}, \mathrm{Et}_{2} \mathrm{O}$, crotonaldehyde, $-78{ }^{\circ} \mathrm{C}$ to $-20{ }^{\circ} \mathrm{C}, 88 \%$; (b) Dess-Martin periodinane, $\mathrm{CH}_{2} \mathrm{Cl}{ }_{2}, \mathrm{r}$.t., $80 \%$; (c) Nysted reagent, $\mathrm{TiCl}_{4}, \mathrm{THF},-20{ }^{\circ} \mathrm{C}$ to $50{ }^{\circ} \mathrm{C}, 63 \%$; (d) TBAF, THF, r.t., $91 \%$; (e) fragment $\mathrm{A}, \mathrm{PPh}_{3}, \mathrm{DIAD}$, THF, $-20{ }^{\circ} \mathrm{C}$ to r.t., $86 \%$; (f) $\mathrm{Cs} 2 \mathrm{CO}_{3}, \mathrm{PhSH}$, acetonitrile, r.t., $86 \%$; (g) (Boc) $)_{2} \mathrm{O}, \mathrm{Et}_{3} \mathrm{~N}$, acetonitrile, r.t.; (h) TBAF, THF, r.t., 79\%(2 steps); (i) Dess-Martin periodinane, $\mathrm{CH}_{2} \mathrm{Cl}_{2}$, r.t., $60 \%$; (j) LiHMDS, methyl propiolate, THF, $-78{ }^{\circ} \mathrm{C}$, $83 \%$; (k) Dess-Martin periodinane, $\mathrm{CH}_{2} \mathrm{Cl}_{2}$, r.t.; (I) BHT, toluene, $80{ }^{\circ} \mathrm{C}, 48 \%$ (2 steps).

we directly subjected 10 to Lewis acid-catalyzed D-A conditions, such as $\mathrm{Et}_{2} \mathrm{AlCl}, \mathrm{ZnCl}_{2}$ and $\mathrm{Sc}(\mathrm{OTf})_{3}$, however, no any D-A product was detected. In addition, heating $\mathbf{1 0}$ in toluene at reflux could not generate the desired D-A product. Afterwards, a more reactive D-A precursor - ketone $\mathbf{1 1}$, which was obtained via DMP oxidation of alcohol 10, was tested under the reaction conditions of $80^{\circ} \mathrm{C}$ without the addition of any catalyst. ${ }^{12}$ Unfortunately, rather than giving the expected [9.3.1] pentadecane skeleton, this reaction produced an undesired [9.2.2] pentadecane skeleton 12 in $48 \%$ yield over two steps from $\mathbf{1 0}$. The structure of $\mathbf{1 2}$ was indirectly identified by the Xray crystal structure of the final product (vide infra). We proposed that the existence of the two newly formed $\mathrm{sp}^{2}$ bridge carbons in the desired D-A product may increase the ring tension of the pentadecane skeleton, thus leading to a relatively high regioselectivity to form the 13-membered [9.2.2] rather than the 12-membered [9.3.1] pentadecane skeleton to reduce the ring tension.

Computational studies were carried out using Gausssian ${ }^{13}$ to understand the regioselectivity of the D-A reaction from $\mathbf{1 1}$ to 12 (See SI). Unfortunately, these DFT calculations suggest that our desired 12_[9.3.1] should be the kinetic product, although the experimentally observed product 12_[9.2.2] is calculated to be thermodynamically more stable. Considering that this D-A reaction is calculated to be irreversible, hence the possibility of transformation from 12_[9.3.1] to 12_[9.2.2] could be excluded. From the results of these calculations, our desired product 12_[9.3.1] should be formed. We envisage the reason for us to only detect 12_[9.2.2] is because 12_[9.3.1] is instable, leading to a trace amount and the difficulty in its detection, and thus the thermodynamically stable 12_[9.2.2] was the only obtained product, indicating that the D-A reaction may be a thermodynamically controlled process.
Considering that the 1,4-diene moiety in D-A product 12 was easily aromatized into a benzene ring, 12 was simply purified via a flash silica gel chromatography and was directly subjected to the hydrogenation conditions. To selectively hydrogenate the trisubstituted $\mathrm{C}=\mathrm{C}$ bond without affecting the tetrasubstituted $\mathrm{C}=\mathrm{C}$ bond, Wilkinson's catalyst and Crabtree's catalyst were investigated. Only Crabtree's catalyst could give trisubstituted $\mathrm{C}=\mathrm{C}$ bond hydrogenated product $\mathbf{1 3}$ in $47 \%$ yield with absolute regio- and stereoselectivities, while the reaction did not proceed under the conditions of Wilkinson's catalyst. This excellent stereoselectivity may attribute to that the steric hindrance of the 6 -face of the boat conformation of the 1,4 diene cyclohexane is larger than that of the $\alpha$-face, thus resulting in the completely facial selective cis-hydrogenation towards the less hindered $\alpha$-face. Then the hydrogenated product 13 was treated with $\mathrm{BF}_{3} \cdot \mathrm{Et}_{2} \mathrm{O}$ at $-30{ }^{\circ} \mathrm{C}$ to remove the Boc protecting group to render amine 14, as well as a trace amount of cascade cyclization product $\mathbf{1 5}$ detected by LC-MS, which inspired us to use other acids to facilitate this cascade reaction. ${ }^{4 \mathrm{n}}$ After extraction of the reaction mixture, amine $\mathbf{1 4}$ was directly subjected to $\mathrm{AcOH}$, stimulating a cascade reaction, and rendering cyclized product $\mathbf{1 5}$ stereo-specifically in 35\% yield. We deduced that this satisfying stereoselectivity may be affected by the stereochemistry at C7. Subsequently, we explored other basic conditions (pyridine and $\mathrm{Et}_{3} \mathrm{~N}$ ) to improve the yield, but no cyclized product 15 was detected. The thioketal group in $\mathbf{1 5}$ was then removed under the promotion of PIFA, affording $\mathbf{1 6}$ as the isomer of lycoplanine A in $83 \%$ yield, the structure of which was unambiguously confirmed by the $X$ ray single crystal diffraction to be a tetracyclic compound with a 3-azabicyclo[6.2.2]dodecane unit (CCDC: 1947434).

In view of the biological activities of Lycopodium alkaloids previously reported, we conducted a biological evaluation of $\mathbf{1 6}$ 


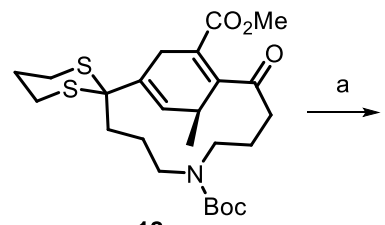

12

||

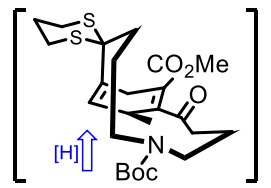

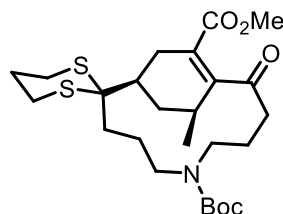

13

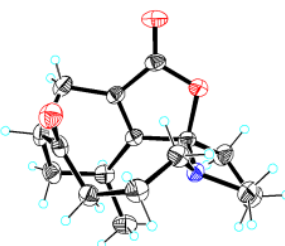

ORTEP of 16
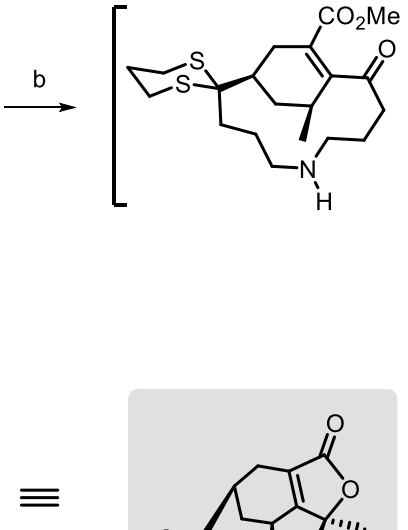

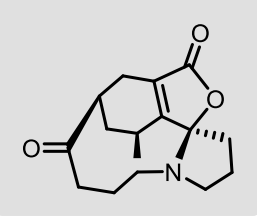

16

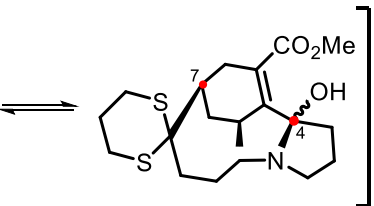

14
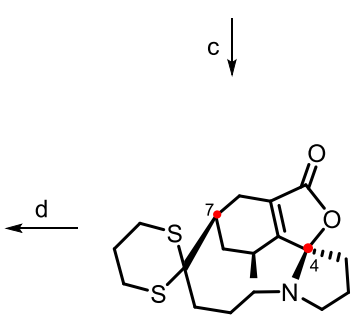

15

Scheme 4 Synthetic route to the isomer of lycoplanine A. Reagents and conditions: (a) Crabtree's catalyst, $\mathrm{H}_{2}, \mathrm{DCE}, 80^{\circ} \mathrm{C}, 47 \%$; (b) $\mathrm{BF} 3 \cdot \mathrm{Et}_{2} \mathrm{O}, \mathrm{DCM},-30{ }^{\circ} \mathrm{C}$; (c) $\mathrm{AcOH}, \mathrm{toluene}^{\circ}, 90^{\circ} \mathrm{C}$, $35 \%$ ( 2 steps); (d) PIFA, MeCN- $\mathrm{H}_{2} \mathrm{O}, 83 \%$. Non-hydrogen atoms are shown as $30 \%$ ellipsoids.

on EeAChE and eqBuChE inhibition. Unfortunately, 16 showed no inhibitory activity on cholinesterase $\left(\mathrm{IC}_{50}>100 \mu \mathrm{M}\right)$.

In conclusion, an isomer of lycoplanine A with an unexpected $6 / 10 / 5 / 5$ tetracyclic skeleton was synthesized during our synthetic route to the natural product lycoplanine A. Diels-Alder reaction and cascade cyclization were employed as the key steps to construct the [9.2.2] pentadecane skeleton and the challenging 1-oxa-6-azaspiro[4.4]nonane spiro moiety, respectively. In addition, we evaluated the preliminarily inhibitory activity of synthesized isomer on cholinesterase but the result was unsatisfactory. Although the final product we synthesized was a complex isomer of lycoplanine $A$, the challenging 1-oxa-6-azaspiro[4.4]nonane spirocenter in lycoplanine $A$ was constructed by an efficient cascade reaction, which provided sufficient experience for the total synthesis of lycoplanine A and natural products with a similar spiro-N,O-acetal moiety. ${ }^{14}$ In addition, this work may be helpful for studying the proposed biogenetic pathway between lycopladine $H$ and lycoplanine A, which may further provide access to synthesize lycopladine $\mathrm{H}$ and lycoplanine $A$. The total synthesis study on lycoplanine $A$ is now underway in our laboratory.

\section{Conflicts of interest}

There are no conflicts to declare.

\section{Acknowledgement}

Financial support was provided by the Recruitment Program of Global Experts (1000 Talents Plan), Gansu Province Science Foundation for Distinguished Young Scholars (20JR5RA304) and the Fundamental Research Funds for the Central Universities (Izujbky-2019-ct08). The authors (B.T. \& L.Y. \& G.M.) acknowledge the financial support from Ministry of Science and Technology of the People's Republic of China under funding scheme National Key R\&D Program of Intergovernmental Key Projects (Grant No. 2018YFE0101700), the National Natural Science Foundation of China (21502101), and the Natural
Science Foundation of Ningbo (2017A610070), the International Doctoral Innovation Centre, Ningbo Education Bureau, Ningbo Science and Technology Bureau, and the University of Nottingham Ningbo China, and acknowledge the University of Nottingham for the use of High Performance Computing Facility.

1 (a) L. S. Liu, Y. L. Zhu, C. M. Yu, Y. Z. Zhou, Y. Y. Han, F. W. Wu and B. F. Qi, Can. J. Chem. 1986, 64, 837; (b) A. P. Kozikowski and W. Tückmantel, Acc. Chem. Res. 1999, 32, 641; and references cited therein.

2 (a) Y. Hirasawa, H. Morita, M. Shiro and J. Kobayashi, Org. Lett. 2003, 5, 3991; (b) J. He, X. Q. Chen, M. M. Li, Y. Zhao, G. Xu, X. Cheng, L. Y. Peng, M. J. Xie, Y. T. Zheng, Y. P. Wang and Q. S. Zhao, Org. Lett. 2009, 11, 1397.

3 (a) W. A. Aver and L. S. Trifonov, Alkaloids Chem \& Pharmacol, 1994, 45, 233; (b) X. Ma and D. R. Gang, Nat. Prod. Rep. 2004, 21, 752.

4 Selected examples for total syntheses of lycopodium alkaloids: (a) C. H. Heathcock, K. M. Smith and T. A. Blumenkopf, J. Am. Chem. Soc. 1986, 108, 5022; (b) X. Linghu, J. J. Kennedy-Smith and F. D. Toste, Angew. Chem., Int. Ed. 2007, 46, 7671; (c) Y. R. Yang, Z. W. Lai, L. Shen, J. Z. Huang, X. D. Wu, J. L. Yin and K. Wei, Org. Lett. 2010, 12, 3430; (d) X. M. Zhang, Y. Q. Tu, F. M. Zhang, H. Shao and X. Meng, Angew. Chem., Int. Ed. 2011, 50, 3916; (e) H. H. Li, X. M. Wang and X. G. Lei, Angew. Chem., Int. Ed. 2012, 51, 491; (f) G. Pan and R. M. Williams, J. Org. Chem. 2012,77, 4801; (g) R. Ding, B. F. Sun and G. Q. Lin, Org. Lett. 2012, 14, 4446; (h) X. M. Zhang, H. Shao, Y. Q. Tu, F. M. Zhang and S. H. Wang, J. Org. Chem. 2012, 77, 8174; (i) N. Itoh, T. Iwata, H. Sugihara, F. Inagaki and C. Mukai, Chem.-Eur. J. 2013, 19, 8665; (j) S. H. Hou, Y. Q. Tu, L. Liu, F. M. Zhang, S. H. Wang and X. M. Zhang, Angew. Chem., Int. Ed. 2013, 52, 11373; (k) J. Zhang, J. B. Wu, B. K. Hong, W. Y. Ai, X. M. Wang, H. H. Li and X. G. Lei, Nat. Commun. 2014, 5, 4614; (I) P. S. Chauhan, J. R. Sacher and S. M. Weinreb, Org. Lett. 2015, 17, 806; (m) X. H. Zhao, Q. Zhang, J. Y. Du, X. Y. Lu, Y. X. Cao, Y. H. Deng and C. A. Fan, J. Am. Chem. Soc. 2017, 139, 7095; (n) C. M. Chen, H. Y. Shiao, B. J. Uang and H. P. Hsieh, Angew. Chem., Int. Ed. 2018, 57, 15572. Reviews for total syntheses of lycopodium alkaloids: (o) C. X. Xiao, L. Cao, J. Wang, Y. L. Miao and H. F. Fan, Chin. J. Org. Chem. 2017, 37, 810; (p) M. Kitajima and H. Takayama, Top. Curr. Chem. 2012, 309, 1.

5 Z. J. Zhang, Y. Nian, Q. F. Zhu, X. N. Li, J. Su, X. D. Wu, J. Yang and Q. S. Zhao, Org. Lett. 2017, 19, 4668. 
6 (a) E. Breuer and S. Zbaida, Tetrahedron, 1975, 31, 499; (b) E. Leete and G. B. Bodem, J. Am. Chem. Soc. 1976, 98, 6321; (c) F. He, Y. Bo, J. D. Altom and E. J. Corey, J. Am. Chem. Soc. 1999, 121, 6771; (d) K. C. Nicolaou, S. M. Dalby, S. Li, T. Suzuki and D. Y.-K. Chen, Angew. Chem., Int. Ed. 2009, 48, 7616; (e) H. Satoh, H. Ueda and H. Tokuyama, Tetrahedron, 2013, 69, 89, (f) S. Sumi, K. Matsumoto, H. Tokuyama and T. Fukuyama, Tetrahedron, 2003, 59, 8571.

7 (a) S. Naud, S. J. Macnaughton, B. S. Dyson, D. J. Woollaston, J. W. P. Dallimore and J. Robertson, Org. Biomol. Chem. 2012, 10, 3506; (b) J. L. Bullington and J. H. Dodd, J. Heterocycl. Chem. 1998, 35, 397.

8 (a) K. C. Nicolaou, S. A. Snyder, T. Montagnon and G. Vassilikogiannakis, Angew. Chem., Int. Ed. 2002, 41, 1668. (b) L. M, Y. J. Hu, J. H. Fan, W. Zhang and C. C. Li, Chem. Soc. Rev. 2020. DOI: $10.1039 / d 0 c s 00365 d$.

9 M. Z. Chen and G. C. Micalizio, J. Am. Chem. Soc. 2012, 134, 1352.

10 (a) W. Tochtermann, S. Bruhn, M. Meints, C. Wolff, E.-M. Peters, K. Peters and H.G.v. Schnering, Tetrahedron, 1995, 51 1623; (b) S. Matsubara, M. Sugihara and K. Utimoto, Synlett, 1998, 1998, 313; (c) C. Aïssa, R. Riveiros, J. Ragot and A. Fürstner, J. Am. Chem. Soc. 2003, 125, 15512.

11 Y. Otsuka, F. Inagaki and C. Mukai. J. Org. Chem. 2010, 75, 3420.

12 L. Z. Liu, J. C. Han, G. Z. Yue, C. C. Li and Z. Yang, J. Am. Chem. Soc. 2010, 132, 13608.

13 Full references for Gaussian please see the supporting information.

14 (a) P. Yates, F. N. MacLachlan, I. D. Rae, M. Rosenberger, A. G. Szabo, C. R. Willis, M. P. Cava, M. Behforouz, M. V. Lakshmikantham and W. Zeiger, J. Am. Chem. Soc. 1973, 95, 7842; (b) E. Breuer and S. Zbaida, Tetrahedron, 1975, 31, 499; (c) F. He, Y. Bo, J. D. Altom and E. J. Corey, J. Am. Chem. Soc 1999, 121, 6771; (d) K. C. Nicolaou, S. M. Dalby, S. Li, T. Suzuki and D. Y. K. Chen, Angew. Chem., Int. Ed. 2009, 48, 7616. 